\title{
Dynamic Bandwidth Allocation in WDM-PON Based Mobile Fronthaul
}

\author{
Chunlei Zhang ${ }^{1,}{ }^{*}$, Gangming Zhao ${ }^{1}$, Chenxi Zhang ${ }^{1}$ and Xunfa Yang ${ }^{2}$ \\ ${ }^{1}$ School of Electronic and Information Engineering, Lanzhou Jiaotong University, Lanzhou 730070, China \\ ${ }^{2}$ Linxia Power Supply Company, State Grid Gansu Electric Power Company, Linxia 731100, China \\ ${ }^{*}$ Corresponding author
}

\begin{abstract}
Wavelength division multiplexing passive optical network (WDM-PON) is an important alternative technique for 5G mobile fronthaul due to the large bandwidth and low latency. Wavelength and bandwidth allocation algorithm is very important in WDM-PON. In this paper, a dynamic wavelength and bandwidth allocation algorithm considering the average traffic difference of the cells in mobile fronthaul is proposed. The algorithm allocates bandwidth with cell average traffic (CAT) under wavelength sharing scheme in WDM-PON based mobile fronthaul. The latency performance of the original static sequence allocation algorithm (SSA) and CAT based dynamic allocation algorithm is compared and analyzed. Simulation results show that the new algorithm can effectively guarantee the QoS of various types of cells in mobile fronthaul.
\end{abstract}

Keywords-WDM-PON; fronthaul; wavelength assignment; bandwidth allocation

\section{INTRODUCTION}

The development of the fifth generation (5G) mobile technology is currently underway to enable a fully connected and mobile society. One of the prominent features of $5 \mathrm{G}$ will be the deployment of small cells [1-4]. Introduction of small-cells into 5G will create an enormous requirement in the transport network to carry massive amounts of data with a minimal delay requirement from hundreds of thousands of cells. In order to support such connectivity, an effective wireless technology and also a cost-effective and greener optical transport solution will be needed. The advancement of wireless networks towards 5G is presenting to optical access networks a technological challenge to deliver high-throughput and low latency.

Passive optical network has a point-to-multipoint architecture, which is naturally suited to cost-effectively carry the common public radio interface (CPRI) traffic, via the sharing of most of the common fiber infrastructure with multiple RRU sites $[2,5]$. There is a clear need for coordination between RAN and PON to simplify the overall access network, reduce the network latency, and improve the network cost-efficiency and power-efficiency as WDM-PON.

In this paper, dynamic bandwidth allocation is studied in mobile fronthaul based on WDM-PON. The second part introduces architecture and bandwidth allocation algorithms of WDM-PON based mobile fronthaul network. The third part describes the proposed dynamic bandwidth allocation algorithm. The simulation results of the proposed algorithm is analyzed in the fourth part. Then we conclude the paper.

\section{WDM-PON BASED MOBILE FRONTHAUL}

\section{A. Mobile Fronthaul Based on WDM-PON}

To meet the great capacity demand for $5 \mathrm{G}$ systems, many small cells will be introduced and deployed with various radio access technologies. Centralized radio access network (C-RAN) architecture will be employed to achieve an efficient operation of small cells and to reduce network cost. Mobile fronthaul connects centralized base band units (BBUs) with remote radio units (RRUs) for purposes such as C-RAN, massive MIMO, and small-cell deployments [1, 2]. Currently, the interface used for mobile fronthaul is primarily based on CPRI. Figure I shows the architecture of mobile fronthaul based on WDM-PON.

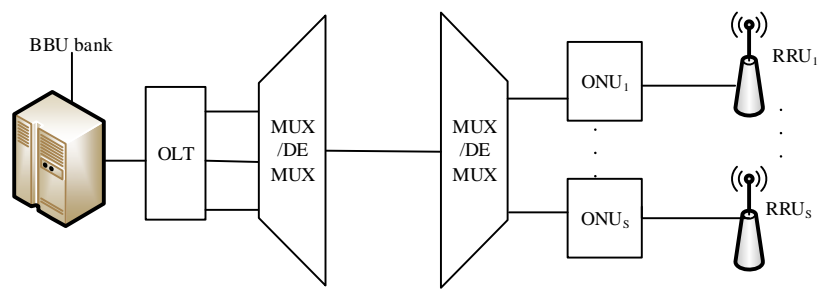

FIGURE I. WDM-PON BASED MOBILE FRONTHAUL.

To meet the stringent latency requirements of $5 \mathrm{G}$ mobile fronthaul, low-latency bandwidth allocation algorithm is needed at OLT. WDM-PON can flexibly allocate wavelength resource to ONUs.

\section{B. Wavelength Bandwidth Allocation Algorithms}

As shown in Figure II, the ideal structure of the WDM-PON system is to allocate a fixed wavelength for each ONU to transmit to the OLT, to achieve point-to-point connection, which is the most ideal WDM-PON wavelength allocation scheme, to ensure effective and reliable communication between OLT and ONUs [3].

In the actual mobile fronthaul, ONUs and RRUs are connected. The number of RRUs is very large, and the light source is expensive. The fixed wavelength allocation mode of WDM-PON deployment costs are too high to be deployed in the mobile fronthaul in large-scale. In addition, in fixed wavelength allocation method the wavelength is fixed for each ONU. The assigned wavelength cannot serve other ONUs. It is difficult to adapt to the flexible mobile fronthaul transmission network scenarios. 


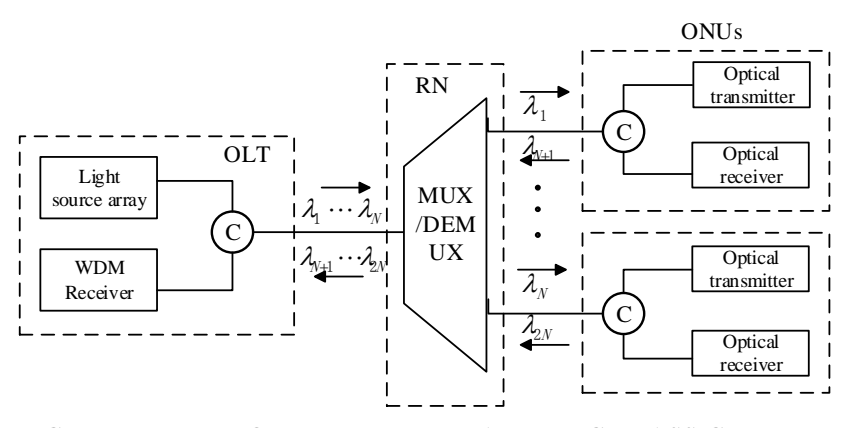

FIGURE II. WDM-PON WITH FIXED WAVELENGTH ASSIGNMENT.

The wavelength sharing WDM-PON system does not fix the wavelength of each ONU, but dynamically allocates bandwidth so that all ONUs of the whole network can share available wavelength. The network is reconfigurable.

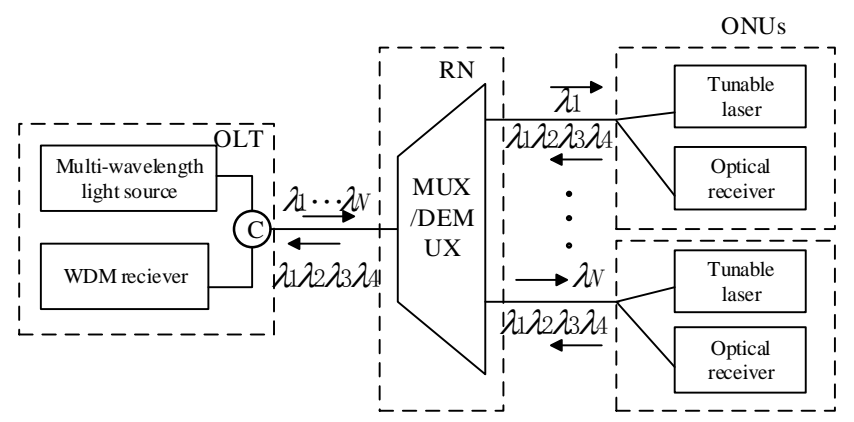

FIGURE III. WDM-PON WITH SHARED WAVELENGTH ASSIGNMENT.

As shown in Figure III is a shared wavelength WDM-PON system, wherein the OLT configure multi-wavelength light source, generating $N$ wavelengths. $N$ ONUs are assigned fixed wavelength to downstream transmission. At ONU tunable lasers are deployed, which can tune to 4 different wavelengths. $N$ ONUs' upstream transmission shares 4 wavelengths. At this time, the system needs to specify the wavelength bandwidth assignment algorithm to allocate the corresponding bandwidth resources for the upstream transmission, and the reasonable wavelength bandwidth allocation algorithm can often improve the QoS of users.

The wavelength bandwidth allocation algorithm of WDMPON system is divided into two categories: static sequential allocation algorithm and dynamic allocation algorithm. The former does not need complex implementation process. It uses principle of "first-come-first-transmission" and "first-requestfirst-allocation" to allocate wavelength and bandwidth. A buffer is set at OLT to cache the downstream data, while a buffer is set at each ONU to cache the upstream data in the algorithm. When the data arrives at the OLT, the OLT inquires whether the current system has a wavelength bandwidth available. If there is available wavelength and bandwidth, the bandwidth is allocated on demand to transmit the data; if there is no available bandwidth, the data goes to the waiting state. ONU seeks the suitable upstream data in its own queue to send according to the length of the received downstream data [4]. Dynamic allocation algorithm is more complex. According to the load difference of actual user or the type of transmission data, the limited bandwidth of the shared wavelength is dynamically allocate to the users. The algorithm can always guarantee the user's QoS.

\section{DYNAMIC BANDWIDTH ALLOCATION ALGORITHM BASED ON CAT}

\section{A. Basic Idea of the Algorithm}

In the actual mobile network, the existing cell can be classified as super busy cell, super idle cell, busy cell, and idle cell. The average traffic of the cell has a large difference [5]. Table I shows resource utilization of all types of cell at the busiest time in one months. Wireless resource utilization has been overflowed in the super busy cell and busy cell. Users in these cells will face more serious network delay as surfing the Internet, especially in the peak of communication. This is not fair. But resource utilization in idle cell and super idle cell is very low, resulting in a serious waste of resources [6]. Therefore, when WDM-PON is used as the mobile fronthaul network scheme, its fixed wavelength allocation method and the static wavelength bandwidth allocation algorithm under shared mode obviously can't satisfy the fair transmission of all kinds of cells and the efficient utilization of the bandwidth resources.

TABLE I. RESOURCE UTILIZATION OF CELLS AT THE BUSIEST TIME IN ONE MONTH

\begin{tabular}{|c|c|c|c|c|}
\hline Cell type & Super busy & busy & idle & Super idle \\
\hline Utilization & $>180 \%$ & $70 \%-180 \%$ & $10 \%-70 \%$ & $<10 \%$ \\
\hline
\end{tabular}

According to the idea of the dynamic wavelength bandwidth allocation algorithm based on service level agreement (SLA) in [7], all kinds of cells are classified into different priority. The super busy cell and the busy cell are listed as high priority cells, correspondingly, the corresponding ONUs are classified as high priority, and the data transmitted by high priority cell is high priority data. Busy cells and super busy cells are classified as low priority, and the corresponding ONUs and transmission data are low priority.

In this algorithm, a polling cycle of the WDM-PON is set. In a polling cycle, the OLT waits and accepts all the ONUs bandwidth request frames. According to priority order, the different cells data is divided into two different priority queue, and calculating the length of two queues, and then allocating the wavelength bandwidth.

\section{B. Description of the Algorithm}

Assuming that there are $N$ ONUs transmit high-priority cell data, $M$ ONUs transmit low-priority cell data. In a polling cycle for wavelength bandwidth allocation, the high priority queue is assigned the transmission bandwidth first, and when the sum of the bandwidth requested by the high priority queue is less than or equal to the sum of the current system available bandwidth, the bandwidth is allocated on demand, if the sum of the current high-priority request bandwidth is greater than the total available bandwidth of the system. According to (1), the corresponding bandwidth is allocated for each high-priority ONU, at which time the system has no remaining available bandwidth, and the low-priority data is discarded for transmission over the next cycle. 


$$
G_{H i}=\left\{\begin{array}{c}
R_{H i}, \sum_{i=1}^{N} R_{H i} \leq B_{\mathrm{a}} \\
\left(R_{H i} / \sum_{i=1}^{N} R_{H i}\right), \sum_{i=1}^{N} R_{H i}>B_{\mathrm{a}}
\end{array}\right.
$$

Equation (1) is the high priority cell bandwidth allocation formula, where $G_{H i}$ is the system-granted bandwidth of the ith high-priority ONU in the current cycle, $R_{H i}$ is the request bandwidth of the high-priority ONU, $B_{a}$ is the available bandwidth of the current system.

After the system bandwidth has been allocated for high priority, if there is any remaining bandwidth, it is continued to allocate for the low priority. There are two cases. When the low priority cell request bandwidth is less than or equal to the remaining available bandwidth, the bandwidth is allocated on demand; otherwise it is allocated by (2).

$$
G_{L \mathrm{j}}=\left\{\begin{array}{c}
R_{L \mathrm{j}}, \sum_{\mathrm{j}=1}^{M} R_{L \mathrm{j}} \leq B_{\mathrm{r}} \\
\left(R_{L \mathrm{j}} / \sum_{\mathrm{j}=1}^{M} R_{L \mathrm{j}}\right), \sum_{\mathrm{j}=1}^{M} R_{L \mathrm{j}}>B_{\mathrm{r}}
\end{array}\right.
$$

Equation (2) is a high priority cell bandwidth allocation formula, where $G_{L j}$ is the system-granted bandwidth of the $j t h$ high-priority ONU in current cycle, $R_{L j}$ is the request bandwidth of the high-priority $\mathrm{ONU}, B_{r}$ is the current available bandwidth of the system.

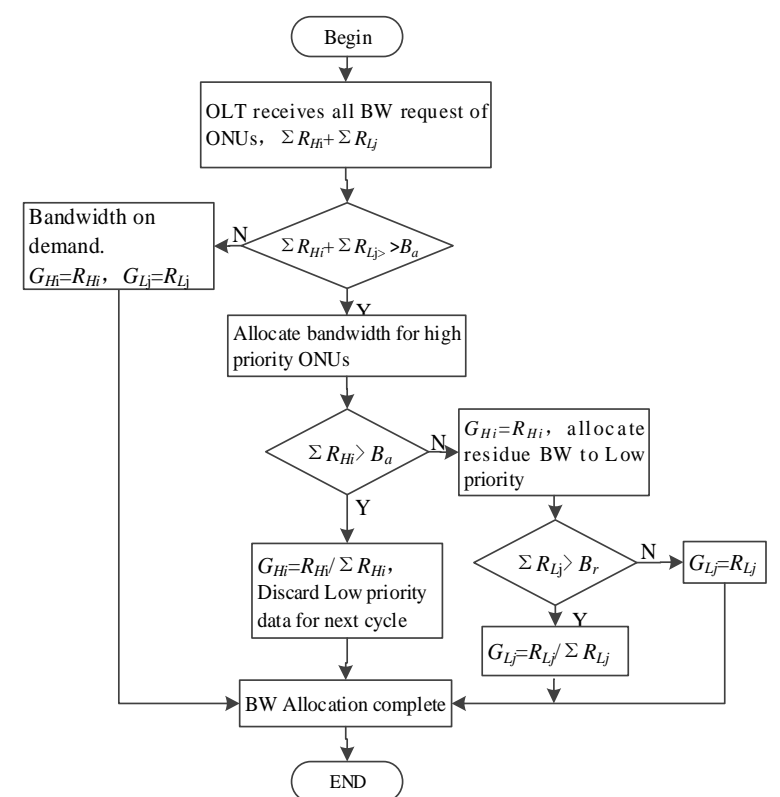

FIGURE IV. FLOWCHART OF THE WAVELENGTH BANDWIDTH ALLOCATION.

The flowchart of dynamic bandwidth allocation process is shown as Figure IV. The procedure is performed at OLT and employs media access control protocols such as the IEEE 802.3ah Multipoint Control Protocol (MPCP) to exchange the necessary control information between the OLT and the ONUs [7]. In MPCP, the OLT uses gate messages to pass information to ONUs about the size of the allocated transport window and the timesheet it transmits, and ONU uses the report message to transmit information about its queue occupancy to the OLT. Authorization scheduling is the allocation of time slots on a supported wavelength channel to allow ONU to efficiently transmit and receive upstream and downstream data and control information. Allocation of bandwidth to the wavelength and time slot allocation, dynamic wavelength time slot allocation is also by the OLT and ONU through the MPCP protocol to achieve. In short, is the first calculation of each ONU allocated bandwidth transmission required time slots, according to priority order, the earliest available wavelength time slot allocated to each ONU transmission data [8].

\section{SimUlation RESUlts OF THE DYNAMIC BANDWIDTH ALLOCATION ALGORITHM}

The CPRI signal specified by ITU-T can achieve the highest rate of $10.137 \mathrm{Gbit} / \mathrm{s}$ through aggregation. In order to better simulate the practice fronthaul, a 10Gbit/s WDM-PON system simulation model is built on the basis of the wavelength sharing system as shown in Figure II. In the system, 16 ONUs share four wavelengths for upstream transmission. The static sequence allocation (SSA) algorithm and the cell average traffic (CAT) based dynamic allocation algorithm are simulated. Due to the stringent delay requirements of the mobile fronthaul networks, the time delay is as the characteristics of QoS parameters for mobile user. The upstream and downstream transmission delay is analyzed by two algorithms respectively.

As shown in Figure V, the downstream average transmission delay is significantly lower than the upstream, and with the increase of network traffic load, the average transmission delay becomes longer. When the load reaches 0.5, the upstream average transmission delay shows a significant increase in the trend, while the downlink average transmission delay presents a slower growth trend. The delay variation reaches the maximum value, which is about $4.3 \mathrm{~ms}$, when the traffic load is 0.9 . The downstream transmission performance is significantly better than upstream transmission with SSA algorithm.

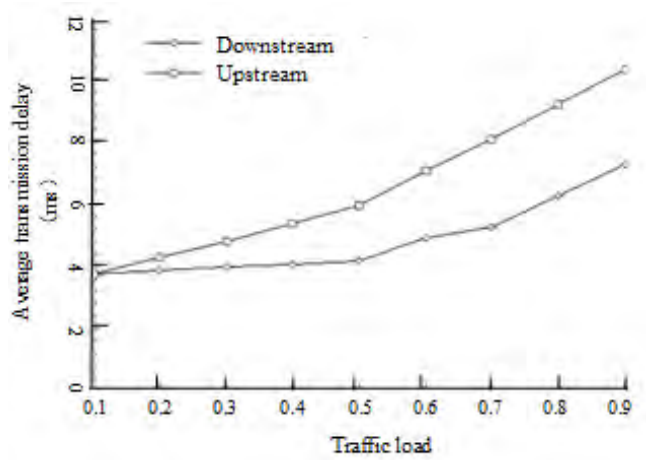

FIGURE V. AVERAGE TIME DELAY OF SSA ALGORITHM 


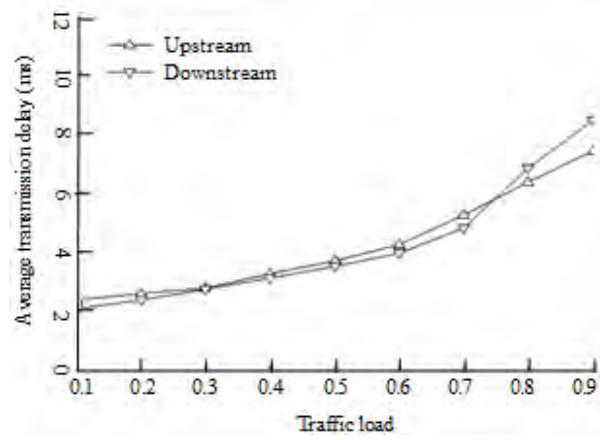

FIGURE VI. AVERAGE DELAY OF CAT BASED ALGORITHM.

The CAT-based dynamic allocation algorithm classifies the ONU-terminal cells according to the request bandwidth of each ONU, and dynamically distributes the wavelength bandwidth by priority, which is based on upstream transmission. As shown in Figure VI, under low traffic load the average transmission delay change trend of the system is basically consistent, all in a slow increase. When the traffic load reaches 0.6 , both upstream and downstream have a rapid increase, the downlink average transmission delay is more obvious. Their time delay variation reaches the maximum, which is about $1 \mathrm{~ms}$, at the same load of 0.9 . The transmission delay performance is basically the same in downstream and upstream.

In the mobile fronthaul, the delay performance is very important. The dynamic allocation algorithm based on CAT can guarantee the fairness of the mobile users' traffic, and the SSA algorithm is obviously not guaranteed. In addition, the average delay performance of a system based on CAT dynamic allocation algorithm is better than that of the SSA algorithm, regardless of the upstream and downstream transmission.

Figure VII a shows the latency performance of a highpriority cell in two algorithms. The latency of the high-priority cell increases slowly with the load increasing, and the delay performance of CAT-based dynamic allocation algorithm is significantly better than the SSA algorithm. The total time delay is $3.3 \mathrm{~ms}$. Figure VIII is the time delay performance of a lowpriority cell in the two algorithms. The delay variation is smaller than the high priority cells, the maximum delay variation is about $1.5 \mathrm{~ms}$. There is a sudden increase at the load of 0.4 in delay curve, this is because the system high priority request bandwidth is greater than the available bandwidth and the low-priority data is transmitted in the next cycle.

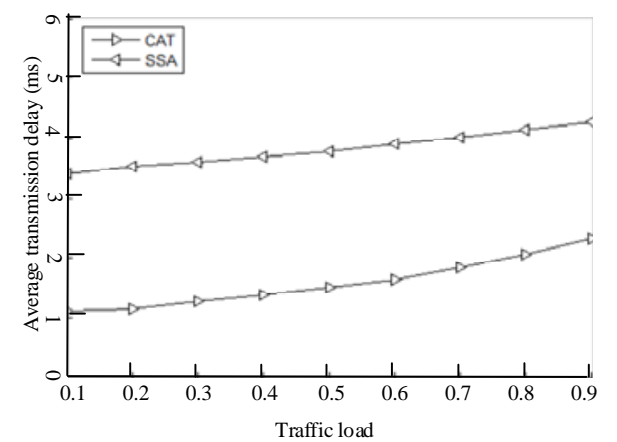

FIGURE VII. AVERAGE DELAY OF HIGH PRIORITY CELLS

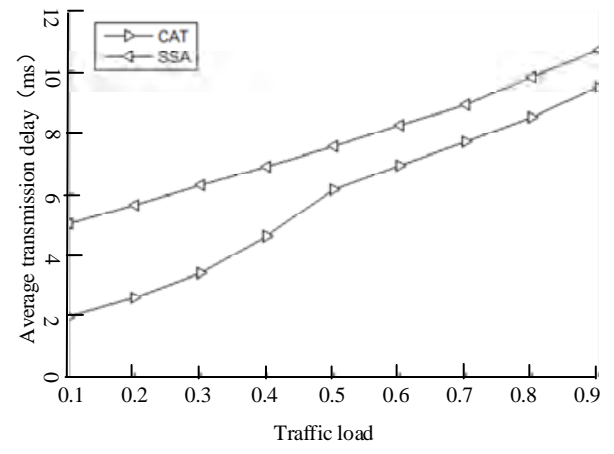

FIGURE VIII. AVERAGE DELAY OF LOW PRIORITY CELLS

\section{CONCLUSION}

With the deep deployment of LTE and the proposal of 5G standard, the mobile fronthaul faces great pressure. WDM-PON system which is secure, high speed, high bandwidth, low delay/jitter and strong reconfigurable both in transmission performance and network upgrade in the future can meet the needs of mobile fronthaul. In this paper, the proposed dynamic bandwidth allocation algorithm based on CAT gives priority to the transmission of super busy cell and busy cell data, avoids the additional delay caused by the overflow of resource utilization, avoids the waste of busy cell and super idle cell resources, and improves the overall delay performance of the network, ensuring the fairness of the transmission of the upstream and downstream effectively improve the QoS of user. With the cost decrease of WDM-PON system and the further improvement of key technology and the increasing of network traffic, WDM-PON will be one of the best scheme for mobile fronthaul.

\section{REFERENCES}

[1] Pizzinat A, Chanclou P, Saliou F, et al. "Things you should know about fronthaul,” Journal of Light-wave Technology, 2015, vol. 33, no. 5, pp.1077-1083.

[2] Keita Takahashi, et al., "NG-PON2 Demonstration with Small Delay Variation and Low Latency for 5G Mobile Fronthaul," European Conference on Optical Communication (ECOC), 2017, pp.1-3.

[3] IEEE 5G, IEEE 5G and Beyond Technology Roadmap White Paper [Online]. Available: https://5g.ieee.org/roadmap, Oct. 2017.

[4] Xiang Liu and Frank Effenberger, "Emerging Optical Access Network Technologies for 5G Wireless [Invited]," Journal of Optical Communication Networks, vol.8, no.12, pp.70-79, Dec. 2016.

[5] B. Skubic, J. Chen, J. Ahmed, B. Mukherjee, "A comparison of dynamic bandwidth allocation for EPON, GPON, and Next-Generation TDM PON,” IEEE Communication Magazine, vol. 47, no. 3, pp.40-48, Mar. 2009

[6] Wenming Xie, Analysis of CPRI Transporting Scheme and Transporting Index,” Design Techniques of Posts and Telecommunicaitons, vol. 11, pp.7-10, 2015.

[7] Song H, Park Y, Banerjee A, et al. "Shared-Wavelength WDM-PON Access Network---Bursty Traffic Accommodation And User-Defined SLA Support,” Optical Internet and Next Generation Network, 2006. COIN-NGNCON 2006. The Joint International Conference, 2006, pp. 383 - 385.

[8] Zhuang W, Zhuang H and Zheng H., "Study on WDM-PON Schemes," International Conference on Business Computing and Global Informatization. IEEE Computer Society, 2011, pp.420-423 